\title{
Diseño de un sistema para la verificación del desarrollo de una competencia del perfil del egresado
}

\author{
Aída M. Ortiz-Solarte, Macarena P. Venegas-Gómez, Mónica X. Espinoza-Concha
}

Introducción. Las universidades determinan el perfil de egreso de sus estudiantes; por tanto, definir programas de estudios conducentes a lograr las competencias de perfil del egresado hace necesario analizar la calidad de éstos. Una forma de alcanzar este objetivo es evidenciando si el plan de estudios de la carrera se organiza de manera que las diferentes asignaturas contribuyen a desarrollar estas competencias gradual y sistemáticamente.

Objetivo. Diseñar un sistema que permita verificar la calidad de un programa de carrera mediante un instrumento que evidencia si las asignaturas contribuyen gradual y sistemáticamente a que el estudiante adquiera las competencias declaradas en el perfil del egresado.

Sujetos y métodos. Se realizó un estudio analítico-explicativo de diseño mixto. Se seleccionó la competencia a evaluar y se confeccionó una encuesta, que permitió cuantificar las competencias y contenidos desarrollados en cada una de las asignaturas estudiadas y determinar las metodologías educativas-evaluativas más utilizadas para cada competencia.

Resultados. Se verificó que el 91\% de las competencias y contenidos necesarios para adquirir la competencia del perfil del egresado se desarrollan con los estudiantes en las asignaturas correspondientes. Además, todas las asignaturas utilizan diversas metodologías educativas-evaluativas que contribuyen al logro de las competencias.

Conclusiones. Este sistema permite verificar que el plan de estudios de la carrera está alineado con la competencia seleccionada del perfil del egresado y, por tanto, se puede aplicar a otros programas de carrera.

Palabras clave. Competencias. Evaluación de aprendizajes. Metodologías educativas. Perfil del egresado. Programa de carrera.

\section{Design of a system for verifying the development of a competence in the profiles of graduates}

Introduction. Graduation profile of students is determined by the universities; therefore, defining study programs leading to achieve graduation profile competencies makes necessary for these programs to undergo a quality analysis. One way to achieve this goal is determining if career curriculum is organized so that different subjects gradually and systematically help develop these skills during undergraduate training.

Aim. To design a system to verify the career program quality with an instrument that shows up if different subjects gradually and systematically help students to acquire the graduation profile stated competencies.

Subjects and methods. An analytical-explanatory, mixed design study was performed in which a graduation profile competition was selected and a survey was designed and applied to quantify the skills and/or contents in each of the subjects studied and besides, help determine what the educational-evaluative methodologies were most commonly used for each competition.

Results. We verified that correspondent subjects developed $91 \%$ of the skills and/or contents needed to acquire the competence profile selected for graduate students. Additionally, all subjects used various educational-evaluative methodologies, so contributing to competencies achievement.

Conclusions. The system verifies curriculum as consistent with selected career competition of graduation profile and therefore can be used in other career programs.

Key words. Assessment of learning. Career program. Graduate profile. Skills. Teaching methodologies.

\section{Introducción}

El aprendizaje es un proceso continuo, sistemático y gradual que debe apuntar al logro de competencias definidas, las cuales deben estar declaradas en el perfil del egresado. Para detectar si se está cumpliendo con esto, resulta clave recabar evidencia que muestre que los programas académicos en los cuales se sustentan las asignaturas del plan de estudios se encuentran alineados con el perfil del egresado,
Universidad Diego Portales. Facultad de Medicina. Escuela de Odontología. Santiago, Chile.

Correspondencia: Prof. Aída Mildred Ortiz Solarte. Universidad Diego Portales. Facultad de Medicina. Escuela de Odontología. Avda. Ejército Libertador, 233. CP 8370068. Santiago (Chile)

E-mail: mildred.ortiz@udp.cl

Conflicto de intereses: No declarado.

Competing interests: None declared.

(c) 2015 FEM 
Tabla I. Relación de las 23 competencias seleccionadas y su asignación a las asignaturas.

Ser capaz de manipular, discriminar y seleccionar materiales e instrumentos adecuados para realizar corte Comprender las propiedades, limitaciones y usos de los biomateriales dentales, su manejo y sus limitaciones

I. Preclínico y Reproducir con cera la anatomía y morfología dentarias.

biomateriales I Clasificar los biomateriales de protección pulpodentinaria y su indicación de acuerdo a estructura, propiedades (físicas, químicas y mecánicas), técnica de manipulación e interacción biológica Manipular los biomateriales de protección pulpodentinaria y aplicarlos sobre dientes artificiales

II. Preclínico y biomateriales II

Utilizar correctamente el equipamiento odontológico básico e instrumental de apoyo en el desarrollo de la atención clínica en odontología Aplicar las normas y elementos de bioseguridad indicados para los diferentes procedimientos realizados en el laboratorio de preclínico Realizar preparaciones biológicas cavitarias para restauraciones directas en materiales plásticos metálicos y plásticos estéticos en dientes de marfilina Manipular correctamente los biomateriales materiales plásticos metálicos y plásticos estéticos, en la restauración de preparaciones biológicas en piezas de marfilina, devolviendo sus características anatómicas

Identificar la localización, extensión y grado de actividad de la patología dental, junto con sus posibles repercusiones en la salud, función y estética del paciente Identificar la etiología, patogenia y anatomía patológica de las enfermedades bucodentales para facilitar su prevención, diagnóstico y tratamiento

III. Cariología Categorizar las lesiones de caries de acuerdo a sus características clínicas, histológicas y bioquímicas

Aplicar un esquema de evaluación de riesgo cariogénico para acercarse al diagnóstico del proceso patológico

Diseñar esquemas preventivos de lesiones de caries y esquemas terapéuticos para la caries

Identificar factores de riesgo cariogénico, propios de ciertos grupos de edad, y establecer pronósticos de los tratamientos

$\begin{array}{ll}\text { IV. Diagnóstico } & \text { Efectuar el procedimiento de toma radiográfica e interpretar las imágenes obtenidas } \\ \text { por imágenes III } & \text { Identificar las distintas técnicas de diagnóstico y seleccionar la adecuada a cada caso }\end{array}$

Seleccionar las opciones de tratamiento que sean adecuadas a las necesidades individuales de cada paciente, compatibles con una terapia actualizada, de calidad y congruentes con una filosofía del cuidado global de su salud bucal

Efectuar la remoción de la caries u otro tipo de tratamientos que tengan el objetivo de eliminarla, empleando técnicas que conserven la viabilidad pulpar Aplicar los conocimientos de la farmacología general y clínica en la práctica odontológica

V. Cínica integral Realizar procedimientos terapéuticos destinados a preservar, establecer o restaurar la forma, función y estética de los dientes, así como la viabilidad de la pulpa dental

Aplicar los conocimientos de las ciencias básicas, competencias y habilidades de las ramas preclínicas y clínicas en la evaluación, planificación y tratamiento de los pacientes

Valorar la ficha clínica y los exámenes complementarios en el marco de documentos de valor ético legal

es decir, si están estructurados de manera que permitan la adquisición de dichas competencias (conocimientos, habilidades y destrezas), requeridas para que los estudiantes puedan seguir avanzando en su aprendizaje y alcanzar finalmente el perfil del egresado.

También se hace necesario determinar si existe una correspondencia entre metodologías, procesos, criterios e instrumentos de evaluación, con el fin de verificar la calidad de los diversos programas de carrera ofertados en la educación superior. Esto permite detectar las carencias en el proceso educativo, específicamente en el área de la programación de los propósitos y metodologías educativas-evaluativas. El objetivo de este trabajo fue idear un sistema para tales efectos en la Facultad de Medicina de la Universidad Diego Portales. Para diseñar este mecanismo, se seleccionó la siguiente competencia del perfil del egresado de la carrera de odontología: 'el cirujano-dentista egresado será capaz de realizar la prevención y tratamiento de la caries dental' [1].

\section{Sujetos y métodos}

Se realizó un estudio analítico-explicativo, de diseño mixto, con una fase cualitativa y otra cuantitativa. Este estudio tuvo una duración de dos años [2], con una primera fase cualitativa que consistió en la selección de la competencia del perfil del egresado a evaluar y se recopiló la información de los conocimientos, habilidades y destrezas involucrados en el desarrollo de dicha competencia, considerando lo descrito en el libro blanco ANECA de la licenciatura de odontología [3], que fue ratificada por el Comité de Currículum de la carrera, que además determinó las principales asignaturas que debían desarrollar estos contenidos (Tabla I).

Para este caso fueron cinco y se denominaron 'asignaturas clave':

- Asignatura clave I. 'Preclínico y biomateriales I', ubicada en el tercer semestre.

- Asignatura clave II. 'Preclínico y biomateriales II', ubicada en el cuarto semestre. 
- Asignatura clave III. 'Cariología', ubicada en el quinto semestre

- Asignatura clave IV. 'Diagnóstico por imágenes III', ubicada en el séptimo semestre.

- Asignatura clave V. 'Clínica integral', ubicada en el undécimo semestre

Se diseñó una encuesta, que fue validada en su constructo y contenido por un grupo de académicos expertos en el área de educación de la Universidad Diego Portales y también mediante un test-retest aplicado a un grupo de docentes que no formaban parte de la muestra [4]. La encuesta consta de cinco ítems: el primero hace referencia a las competencias y contenidos que, de acuerdo con el Comité de Currículum de la Facultad de Medicina de la Universidad Diego Portales, deben tratarse en cada una de las asignaturas clave y por lo tanto deberían incluirse en sus respectivos programas. El segundo indaga sobre las metodologías educativas más utilizadas; el tercero, sobre la existencia de procesos de evaluación; el cuarto, sobre la definición de los criterios de evaluación explicitados en los programas de asignatura, y el quinto, sobre los diversos instrumentos de evaluación aplicados. Esta encuesta era la misma para todas las asignaturas, exceptuando las competencias y contenidos asignados a cada una de ellas (Fig. 1).

La muestra estuvo constituida por seis académicos para cada una de las asignaturas clave; por tanto, la encuesta fue respondida por 30 docentes simultáneamente. Para responderla, se les proporcionó instrucciones y el programa de su respectiva asignatura.

Las variables a estudiar fueron cinco:

- Contenidos: numero de competencias y contenidos declarados por cada asignatura que coinciden con las determinadas por el Comité de $\mathrm{Cu}$ rrículum.

- Metodologías educativas utilizadas por las asignaturas.

- Procesos de evaluación: presencia de procesos declarados para evaluar las competencias y contenidos consultados.

- Criterios de evaluación: existencia de criterios declarados para evaluar las competencias y contenidos consultados.

- Instrumentos de evaluación utilizados para evaluar las competencias y contenidos.

Para cada variable se establecieron tres categorías: óptimo, suficiente e insuficiente. Para determinar las categorías se utilizaron los criterios presentados en la tabla II.
Figura 1. Modelo de la encuesta utilizada. 1: totalmente en desacuerdo; 2 : en desacuerdo; 3: indiferente; 4: de acuerdo; 5: totalmente de acuerdo.

\begin{tabular}{|c|c|c|c|c|c|c|}
\hline \multicolumn{3}{|c|}{$\begin{array}{l}\text { 1. La asignatura declara el desarrollo de las siguientes competencias en el nivel correspondiente } \\
\text { según su ubicación en la malla curricular (principiante, novato, competente). Marque con una X } \\
\text { bajo el número que más lo identifica. Debe justificar su opción }\end{array}$} & 12 & 23 & 4 & 5 \\
\hline \multicolumn{3}{|l|}{ Competencia 1} & & & & \\
\hline \multicolumn{7}{|l|}{ Justifique su respuesta: } \\
\hline \multicolumn{3}{|l|}{ Competencia 2} & & & & \\
\hline \multicolumn{7}{|l|}{ Justifique su respuesta: } \\
\hline \multicolumn{7}{|c|}{$\begin{array}{l}\text { II. Para las siguientes competencias, indicar la(s) metodologia(s) utilizada(s). } \\
\text { Escriba una o más letras de acuerdo a las metodologías utilizadas }\end{array}$} \\
\hline \multicolumn{7}{|l|}{ Competencia 1} \\
\hline \multicolumn{7}{|l|}{ Competencia 2} \\
\hline $\begin{array}{l}\text { A. Clase expositiva } \\
\text { D. Talleres } \\
\text { G. Estudio de casos } \\
\text { J. Resolución de problemas }\end{array}$ & $\begin{array}{l}\text { B. Seminarios } \\
\text { E. Role play } \\
\text { H. Prácticas externas } \\
\text { K. Otros (indique cuál) }\end{array}$ & $\begin{array}{l}\text { C. Aprendizajo basadc } \\
\text { F. Simulaciones } \\
\text { 1. Tutorías }\end{array}$ & lomas & $\overline{s(A)}$ & & \\
\hline \multicolumn{3}{|c|}{$\begin{array}{l}\text { III. La asignatura realiza procesos de evaluación de las siguientes competencias. } \\
\text { Marque con una } \mathrm{X} \text { bajo el número que más lo identifica. Debe justificar su opción }\end{array}$} & 12 & 23 & 3 & 5 \\
\hline \multicolumn{3}{|l|}{ Competencia 1} & & & & \\
\hline \multicolumn{7}{|l|}{ Justifique su respuesta: } \\
\hline \multicolumn{3}{|l|}{ Competencia 2} & & & & \\
\hline \multicolumn{7}{|l|}{ Justifique su respuesta: } \\
\hline \multicolumn{3}{|c|}{$\begin{array}{l}\text { IV. ¿Existen criterios de evaluación específicos para evaluar la competencia? } \\
\text { Marque con una } X \text { bajo el número que más lo identifica. Debe justificar su opción }\end{array}$} & 12 & 23 & 4 & 5 \\
\hline \multicolumn{3}{|l|}{ Competencia 1} & & & & \\
\hline \multicolumn{7}{|l|}{ Justifique su respuesta: } \\
\hline \multicolumn{3}{|l|}{ Competencia 2} & & & & \\
\hline \multicolumn{6}{|l|}{ Justifique su respuesta: } & \\
\hline \multicolumn{6}{|c|}{$\begin{array}{l}\text { V. Para las siguientes competencias, indique el(los) instrumento(s) de evaluación utilizado(s). } \\
\text { Escriba una o más letras de acuerdo a los instrumentos utilizados }\end{array}$} & \\
\hline \multicolumn{7}{|l|}{ Competencia 1} \\
\hline \multicolumn{6}{|l|}{ Competencia 2} & \\
\hline A. $360^{\circ}$ & B. Lista de control & C. Escalas de valoració & & & & \\
\hline D. Rúbricas & E. Diario de registro & F. Registros anecdótico & & & & \\
\hline $\begin{array}{l}\text { G. Registros observacionales } \\
\text { J. Otros (indique cuáles) }\end{array}$ & H. Resolución de casos & 1. Pruebas escritas & & & & \\
\hline
\end{tabular}

La segunda fase de la investigación (cuantitativa) correspondió a la aplicación de la encuesta y el análisis de ésta para la obtención de resultados.

\section{Resultados}

En la tabla III se muestra la categoría obtenida (óptimo, suficiente, insuficiente) por cada una de las asignaturas clave en relación a las variables en estudio de acuerdo con la opinión de los académicos encuestados. La asignatura clave IV es la única que obtiene la categoría de 'óptimo' en todas las variables, por lo que presenta alineación entre ellas. Las asignaturas clave I y II presentan categoría de 'suficiente' en criterios y procesos de evaluación, respectivamente, y las asignaturas III y $\mathrm{V}$ muestran deficiencias en las variables de competencias declaradas, criterios y procesos de evaluación. 
Tabla II. Criterios para determinar las categorías.

\begin{tabular}{|c|c|c|c|}
\hline & Óptimo & Suficiente & Insuficiente \\
\hline Contenidos & $\begin{array}{l}\text { Todos los encuestados responden estar } \\
\text { de acuerdo o totalmente de acuerdo } \\
\text { con que la competencia consultada } \\
\text { es desarrollada por la asignatura }\end{array}$ & $\begin{array}{l}\text { Hasta dos encuestados responden no } \\
\text { estar de acuerdo o totalmente de acuerdo } \\
\text { con que la competencia consultada } \\
\text { es desarrollada por la asignatura }\end{array}$ & $\begin{array}{l}\text { Tres o más encuestados responden no } \\
\text { estar de acuerdo o totalmente de acuerdo } \\
\text { con que la competencia consultada } \\
\text { es desarrollada por la asignatura }\end{array}$ \\
\hline $\begin{array}{l}\text { Metodologías } \\
\text { educativas }\end{array}$ & $\begin{array}{l}\text { La asignatura utiliza, además } \\
\text { de la clase expositiva o seminario, } \\
\text { dos o más metodologías diferentes }\end{array}$ & $\begin{array}{l}\text { La asignatura utiliza, además } \\
\text { de la clase expositiva o seminario, } \\
\text { una metodología diferente }\end{array}$ & $\begin{array}{l}\text { La asignatura sólo utiliza la } \\
\text { clase expositiva o seminario }\end{array}$ \\
\hline $\begin{array}{l}\text { Criterios de } \\
\text { evaluación }\end{array}$ & $\begin{array}{l}\text { Todos los encuestados responden estar } \\
\text { de acuerdo o totalmente de acuerdo } \\
\text { con que existen criterios de evaluación } \\
\text { declarados para la competencia } \\
\text { consultada }\end{array}$ & $\begin{array}{l}\text { Hasta dos de los encuestados } \\
\text { responden no estar de acuerdo o } \\
\text { totalmente de acuerdo con que existen } \\
\text { criterios de evaluación declarados para } \\
\text { la competencia consultada }\end{array}$ & $\begin{array}{l}\text { Tres o más de los encuestados } \\
\text { responden no estar de acuerdo o } \\
\text { totalmente de acuerdo con que existen } \\
\text { criterios de evaluación declarados para } \\
\text { la competencia consultada }\end{array}$ \\
\hline $\begin{array}{l}\text { Procesos de } \\
\text { evaluación }\end{array}$ & $\begin{array}{l}\text { Todos los encuestados responden estar } \\
\text { de acuerdo o totalmente de acuerdo } \\
\text { con que existen procesos de evaluación } \\
\text { declarados para la competencia } \\
\text { consultada }\end{array}$ & $\begin{array}{l}\text { Hasta dos de los encuestados } \\
\text { responden no estar de acuerdo o } \\
\text { totalmente de acuerdo con que existen } \\
\text { procesos de evaluación declarados para } \\
\text { la competencia consultada }\end{array}$ & $\begin{array}{l}\text { Tres o más de los encuestados } \\
\text { responden no estar de acuerdo o } \\
\text { totalmente de acuerdo con que existen } \\
\text { procesos de evaluación declarados para } \\
\text { la competencia consultada }\end{array}$ \\
\hline $\begin{array}{l}\text { Instrumentos } \\
\text { de evaluación }\end{array}$ & $\begin{array}{l}\text { La asignatura utiliza, además } \\
\text { de las pruebas escritas, dos o más } \\
\text { instrumentos diferentes de evaluación }\end{array}$ & $\begin{array}{l}\text { La asignatura utiliza, además de } \\
\text { las pruebas escritas, un instrumento } \\
\text { diferente de evaluación }\end{array}$ & $\begin{array}{l}\text { La asignatura utiliza sólo las pruebas } \\
\text { escritas como instrumento de evaluación }\end{array}$ \\
\hline
\end{tabular}

Tabla III. Resumen de la categoría obtenida para cada una de las asignaturas clave en relación a las variables en estudio.

\begin{tabular}{lccccc}
\hline & Asignatura clave 1 & Asignatura clave 2 & Asignatura clave 3 & Asignatura clave 4 & Asignatura clave 5 \\
\hline Competencias declaradas & Óptimo & Óptimo & Insuficiente & Óptimo & Insuficiente \\
\hline Criterios de evaluación & Suficiente & Óptimo & Insuficiente & Óptimo & Suficiente \\
\hline Procesos de evaluación & Óptimo & Suficiente & Suficiente & Óptimo & Insuficiente \\
\hline Metodologías docentes & Óptimo & Óptimo & Óptimo & Óptimo & Óptimo \\
\hline Instrumentos de evaluación & Óptimo & Óptimo & Óptimo & Óptimo & Óptimo
\end{tabular}

En cuanto a las metodologías educativas e instrumentos de evaluación, se evidencia que todas las asignaturas utilizan metodologías e instrumentos variados para la construcción de la competencia en estudio (Figs. 2 y 3 ).

Considerando como $100 \%$ las 23 competencias seleccionadas y distribuidas en las cinco asignaturas clave, los académicos determinaron que un $91 \%$ de éstas -es decir, 21 competencias-, estaban incluidas en los programas de las mismas. En las asignaturas clave III y $\mathrm{V}$ es donde se evidencia la falta de desarrollo de algunos conceptos y competencias que les atañen (Tabla IV).

\section{Discusión}

Los resultados de este estudio avalan de forma clara que el instrumento elaborado para el mecanismo de análisis permite verificar si una competencia del perfil del egresado se ha desarrollado de forma gradual y sistemática en el trascurso de un plan de carrera, considerando si las asignaturas clave para dicha competencia entregan o desarrollan las competencias y contenidos que les atañen.

Además, este instrumento permite recopilar información sobre metodologías educativas y de evaluación más utilizados. 
En este estudio se comprobó que la competencia seleccionada del perfil del egresado de la carrera de odontología se construye gradual y sistemáticamente en el transcurso del plan de estudio, es decir, las competencias y contenidos trabajados por las asignaturas analizadas coinciden con las requeridas para alcanzar la competencia en estudio.

El hecho de que exista un $91 \%$ de cumplimiento de las competencias desarrolladas con las establecidas como necesarias responde a la ubicación estratégica de las asignaturas en el plan de la carrera, de manera que permitan una integración tanto vertical (articulación o relación entre asignaturas que tradicionalmente se enseñan en diferentes ciclos) como horizontal (articulación entre disciplinas o asignaturas tradicionalmente enseñadas en el mismo año o ciclo del plan de estudios) de disciplinas básicomédicas y odontológicas, considerando que las nuevas tendencias en educación médica indican que el desarrollo de las competencias psicomotrices se debe iniciar desde el primer año de la carrera [5-7].

A partir del año 2000 existe una tendencia mundial para que la formación profesional se oriente al cumplimiento de competencias, lo que ha llevado a que las instituciones de educación replanteen los diseños curriculares tradicionales para garantizar la formación de profesionales que cumplan con los requerimientos adecuados para el ejercicio profesional. Esto necesariamente implica disponer de una combinación de las diferentes metodologías educativas y evaluativas de acuerdo con las características individuales y los contextos socioculturales de la institución y las necesidades de los estudiantes en los diferentes niveles cursados [6,8,9].

Se debe considerar que las competencias articulan conocimiento conceptual, procedimental y actitudinal, pero el hecho de acumular conocimientos no implica ser competente necesariamente. El ser competente implica un paso más: supone, de todo el acervo de conocimiento que uno posee o puede acceder, seleccionar el que resulta pertinente en aquel momento o situación para poder resolver el problema en cuestión. Por tal motivo, es necesario contar con diversas metodologías pedagógicas para facilitar la adquisición de los conocimientos y su integración por parte de los estudiantes, así como también definir los criterios que se utilizarán para evaluar estos aprendizajes, lo que permite seleccionar los métodos e instrumentos adecuados para cada situación. De esta manera se trabaja en la coherencia entre el proceso educativo y el proceso evaluativo $[10,11]$.

El mecanismo utilizado permitió determinar que en las asignaturas clave evaluadas se emplean dife-
Figura 2. Metodologías educativas usadas por las asignaturas.

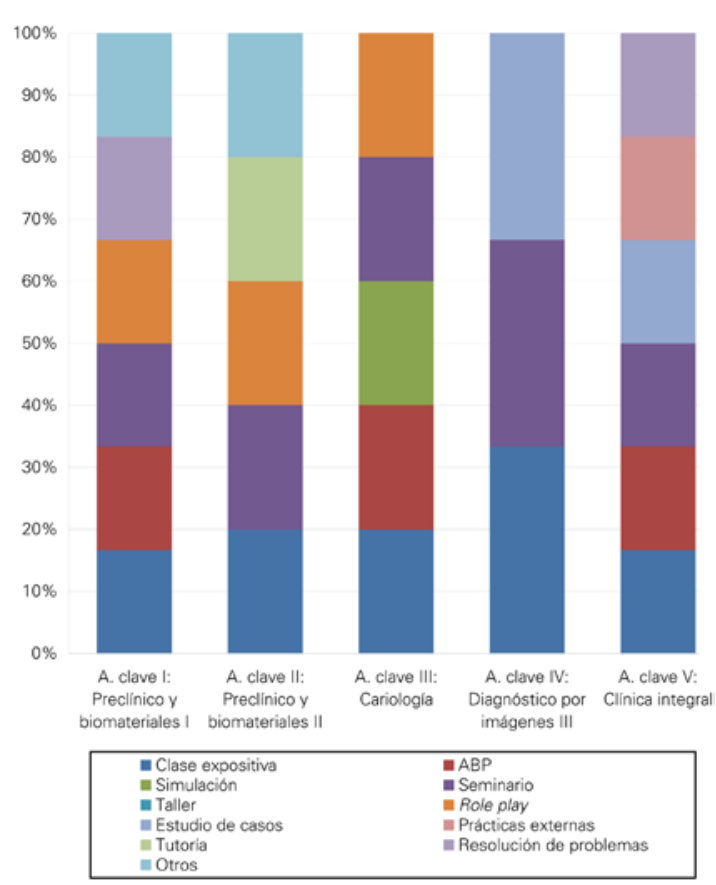

Figura 3. Instrumentos de evaluación utilizados en las asignaturas

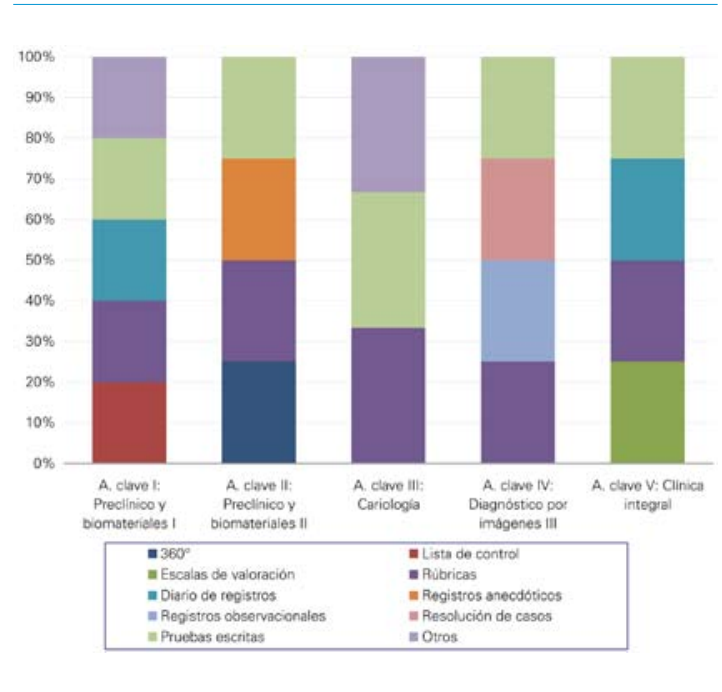

rentes metodologías educativas, como clases expositivas, seminarios, talleres y prácticas simuladas, garantizando que los estudiantes puedan integrar los diferentes saberes. Todas ellas han definido en sus respectivos programas los criterios de evaluación 
Tabla IV. Porcentaje de las competencias desarrolladas por las asignaturas clave que coinciden con las seleccionadas para adquirir las competencias del perfil del egresado.

\begin{tabular}{|c|c|c|c|c|c|c|c|}
\hline & & P1 & P2 & P3 & 11 & 12 & 13 \\
\hline \multirow{5}{*}{$\begin{array}{l}\text { Asignatura } \\
\text { clave } 1\end{array}$} & Competencia 1 & 1 & 1 & 1 & 1 & 1 & 1 \\
\hline & Competencia 2 & 1 & 1 & 1 & 1 & 1 & 1 \\
\hline & Competencia 3 & 1 & 1 & 1 & 1 & 1 & 1 \\
\hline & Competencia 4 & 1 & 1 & 1 & 1 & 1 & 1 \\
\hline & Competencia 5 & 1 & 1 & 1 & 1 & 1 & 1 \\
\hline \multirow{4}{*}{$\begin{array}{l}\text { Asignatura } \\
\text { clave } 2\end{array}$} & Competencia 1 & 1 & 1 & 1 & 1 & 1 & 1 \\
\hline & Competencia 2 & 1 & 1 & 1 & 1 & 1 & 1 \\
\hline & Competencia 3 & 1 & 1 & 1 & 1 & 1 & 1 \\
\hline & Competencia 4 & 1 & 1 & 1 & 1 & 1 & 1 \\
\hline \multirow{6}{*}{$\begin{array}{l}\text { Asignatura } \\
\text { clave } 3\end{array}$} & Competencia 1 & 1 & 1 & 1 & 0 & 0 & 0 \\
\hline & Competencia 2 & 1 & 1 & 1 & 1 & 1 & 1 \\
\hline & Competencia 3 & 1 & 1 & 1 & 1 & 1 & 1 \\
\hline & Competencia 4 & 1 & 1 & 0 & 0 & 0 & 1 \\
\hline & Competencia 5 & 1 & 1 & 1 & 0 & 0 & 1 \\
\hline & Competencia 6 & 1 & 1 & 1 & 1 & 1 & 1 \\
\hline \multirow{2}{*}{$\begin{array}{l}\text { Asignatura } \\
\text { clave } 4\end{array}$} & Competencia 1 & 1 & 1 & 1 & 1 & 1 & 1 \\
\hline & Competencia 2 & 1 & 1 & 1 & 1 & 1 & 1 \\
\hline \multirow{6}{*}{$\begin{array}{l}\text { Asignatura } \\
\text { clave } 5\end{array}$} & Competencia 1 & 0 & 1 & 1 & 1 & 1 & 1 \\
\hline & Competencia 2 & 1 & 1 & 1 & 1 & 1 & 1 \\
\hline & Competencia 3 & 0 & 1 & 1 & 0 & 0 & 1 \\
\hline & Competencia 4 & 1 & 1 & 1 & 1 & 1 & 1 \\
\hline & Competencia 5 & 0 & 1 & 1 & 1 & 1 & 1 \\
\hline & Competencia 6 & 1 & 1 & 1 & 1 & 1 & 1 \\
\hline Total & 21 (91\%) & 20 (87\%) & 23 (100\%) & 22 (96\%) & 19 (83\%) & 19 (83\%) & $22(96 \%$ \\
\hline
\end{tabular}

1: 'muy de acuerdo' o 'de acuerdo'; P1, P2, P3: profesores o docentes de asignaturas; 11, 12, 13: investigadores.

que se utilizarán, así como los procesos e instrumentos para cada una de las instancias evaluadas. Los instrumentos más utilizados para este caso fue- ron rúbricas y pruebas escritas de selección múltiple y respuesta única.

Los mecanismos orientados a la mejora de la calidad de la educación universitaria deben incluir un conjunto de acciones. Una de estos mecanismos es la auditoría académica, que se sustenta en la evaluación interna (o autoevaluación) y la evaluación externa. Otro método es el seguimiento de los egresados y su posicionamiento en el campo laboral, que permite validar los planes y programas y además perfilar alternativas para estar a la altura de las necesidades del mercado laboral. Estos mecanismos por separado no permiten intervenir y hacer las correcciones a tiempo.

Por tal motivo, se debe considerar la aplicación de métodos combinados que faciliten la evaluación del proceso formativo y que entreguen información en distintos tiempos para poder desarrollar las medidas necesarias que permitan corregir aquellos aspectos que no están siendo bien evaluados [12-15].

Finalmente, los resultados obtenidos tras la aplicación de este mecanismo demuestran la falta de alineación de algunas asignaturas clave con las variables en estudio en distintas categorías, por lo que se sugiere aplicarlo a otras competencias del perfil del egresado.

\section{Bibliografía}

1. Facultad de Medicina, Universidad Diego Portales; 2013. Odontología: perfil del egresado. URL: http://www.udp.cl/ facultades_carreras/medicina/odontologia_perfil_egreso.aspp. [24.10.2013].

2. López A. Metodología de la investigación. 1 ed. Valparaíso: Universidad de Playa Ancha de Ciencias de la Educación; 2006

3. Argemí J, Sánchez A, García A, Rodríguez F. Libro blanco del título de Grado de Odontología. Madrid: Agencia Nacional de Evaluación de la Calidad y Acreditación; 2004.

4. Hernández R, Fernández C, Baptista P. Metodología de la investigación. 3 ed. México DF: McGraw-Hill; 2003.

5. Calatrava LA. Educación por competencias en odontología. Acta Odontológica Venezolana 2010. URL: http://www. actaodontologica.com/ediciones/2010/1/pdf/art16.pdf. [24.10.2013].

6. Sanz M, Antoniazzi J. Libro del Proyecto Latino Americano de Convergencia en Educación Odontológica (PLACEO). Sao Paulo: Artes Médicas; 2010.

7. Snyman WD, Kroon J. Vertical and horizontal integration of knowledge and skills -a working model. Eur J Dent Educ 2005; 9: 26-31.

8. Palés J, Gual A. Recursos educativos en ciencias de la salud. Educ Med 2004; 7 (Supl 1): 4-9.

9. Aguilera X, González C, Guerrero A, Bedregal P, Milosavljevic V, Rivera M, et al. Los objetivos sanitarios de la década 2000-2010. El Vigía 2002. URL: http://epi.minsal.cl/epi/html/elvigia/ vigia15.pdf. [24.10.2013].

10. Boone WJ, McWhorter AG, Seale NS. Purposeful assessment techniques (PAT) applied to an OSCE-based measurement of competencies in a pediatric dentistry curriculum. J Dent Educ 2001; 65: 1232-7.

11. Cano M. La evaluación por competencias en la educación superior. Profesorado. Revista de Currículum y Formación 
del Profesorado 2008. URL: http://www.ugr.es/ recfpro/ rev123COL1.pdf. [24.10.2013].

12. Aldana G, Morales F, Aldana J, Sabogal F, Ospina A. Seguimiento a egresados. Su importancia para las instituciones de educación superior. Revista Teoría y Praxis Investigativa 2008; 3: 61-65. URL: http://dialnet.unirioja.es/servlet/ articulo? codigo $=3701001$. [24.10.2013].

13. Betancourt F, De Luna H, Hernández JL. Política educativa, seguimiento de egresados y mercado laboral en las instituciones de educación superior en México. Cuadernos de Educación y Desarrollo 2010. URL: http://www.eumed.net/rev/ced/21/ nls.htm. [24.10.2013].
14. Pires S, Lemaitre M J. Sistemas de acreditación y evaluación de la educación superior en América Latina y el Caribe. Venezuela: IESALC-UNESCO; 2008. URL: http://www. unesco.org.ve/dmdocuments/biblioteca/libros/capitulos/ TENDENCIAS/TEND-CAP 8.pdf. [29.10.2013].

15. González L, Ayarza H. Calidad, evaluación institucional y acreditación en la educación superior en la región latinoamericana y el Caribe. Santiago de Chile: Cinda; 1997. URL: http://biblioteca.uahurtado.cl/ujah/Reduc/pdf/pdf/8184.pdf. [29.10.2013] 